\title{
Backfire Radiation from a Monofilar Helix with a Small Ground Plane
}

\author{
HISAMATSU NAKANO, SENIOR MEMBER, IEEE, JUNJI YAMAUCHI, MEMBER, IEEE, AND HIROAKI MIMAKI
}

\begin{abstract}
Numerical analysis of a helical antenna is made in the presence of a finite grid ground plane. It is found that the transition from forwardfire radiation to backfire radiation occurs as the ground plane diameter is reduced to the order of the helix diameter. The investigation of a backfire helix with a small ground plane shows that a circularly polarized wave is radiated over a frequency range of more than 1 to 1.3 . An application of the backfire helix to a primary feed for a paraboloidal reflector is also described, in which the far-field pattern is evaluated by the physical optics approximation.
\end{abstract}

\section{INTRODUCTION}

A MONOFILAR HELICAL antenna with a ground plane usually operates in the forward endfire mode, or the axial mode, when the circumference of the helix is of the order of one wavelength [1]. Experimental surveys of the axial-mode helical antenna have fully been done by King and Wong [2], who succeed in giving design information. Theoretical surveys of this antenna are also found in [3] and [4], in which the ground plane situated under a helical wire is assumed to be infinite in size, and the image theory is used. There have, however, been few numerical analyses taking account of the effects of a finite ground plane on the radiation field [5].

First, the present paper deals with the effects of size of a ground plane on the radiation characteristics. The ground plane is made of wire grids, and the current distribution is evaluated using the numerical technique, which yields the reasonably accurate radiation field. It is theoretically found that as the ground plane diameter is decreased, the backward radiation becomes dominant.

Secondly, consideration is given to the radiation characteristics of a backfire helical antenna [6], [7] with a small wiregrid ground plane. The analysis covers the helices of pitch angles of $6^{\circ}$ to $18^{\circ}$ over a frequency range of 7 to $13 \mathrm{GHz}$. Theoretical data show that the monofilar backfire helix has advantages of small cross section and easy generation of a circularly polarized wave.

The advantages of the backfire helix encourage us to use this antenna as a primary feed of a paraboloidal reflector [8]. Although other types of a backfire helix are used as a feed for a reflector [9], [10], the theoretical study has not been made. The last part of this paper deals with the analysis of the paraboloidal reflector antenna fed by a monofilar backfire helix. The motivation of this study comes from the development of a direct receiving antenna of the broadcasting satellite

Manuscript received September 1, 1987

The authors are with the College of Engineering, Hosei University, 3-7-2 Kajino-cho, Koganei, Tokyo 184, Japan.

IEEE Log Number 8822569.

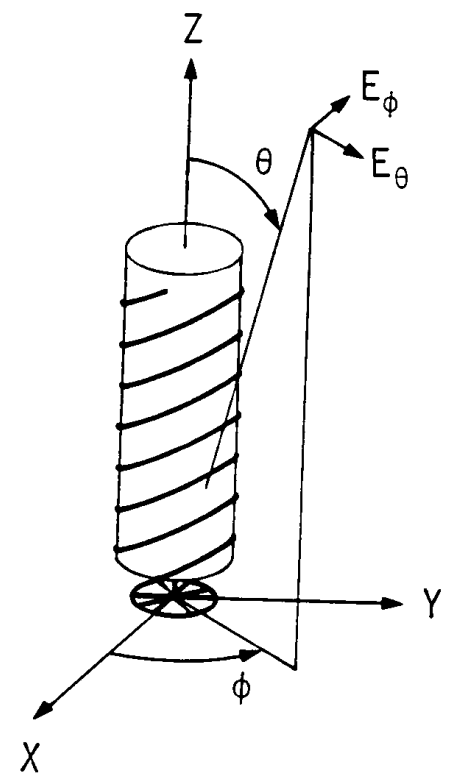

Fig. 1. Configuration and coordinate system.

TV programs (DBS) in Japan. Calculations show the phase center location of the backfire helix and the far-field pattern. The latter is evaluated by the physical optics approximation [11].

\section{Effects of Wire-Grid Ground Plane Diameter}

Fig. 1 shows the configuration and coordinate system of a helical antenna to be considered here. The ground plane has a diameter range of $0.29 \lambda$ to $0.60 \lambda$, where $\lambda$ is the free-space wavelength. The number of helical turns $n$, circumference of helical cylinder $C$, pitch angle $\alpha$, and wire radius $\rho$ are fixed to be $n=7, C=1 \lambda, \alpha=12.5^{\circ}$, and $\rho=0.012 \lambda$, respectively. The ground plane is made of wire grids.

Fig. 2 shows the behavior of the current distribution and radiation pattern when the size of the ground plane is changed. In the numerical analysis we apply a piecewise sinusoidal Galerkin method [11], [12] to an evaluation of the current distribution, by which the radiation fields are calculated. The applied voltage is $1 \mathrm{~V}$.

In Fig. 2(a), where the diameter of the ground plane is $d=$ $0.60 \lambda$, the current distribution has two distinct regions; one is a decaying region starting from the input toward the first minimum shown by an arrow, and the other is a surface wave region after the first minimum. The relative phase velocity $p$ 


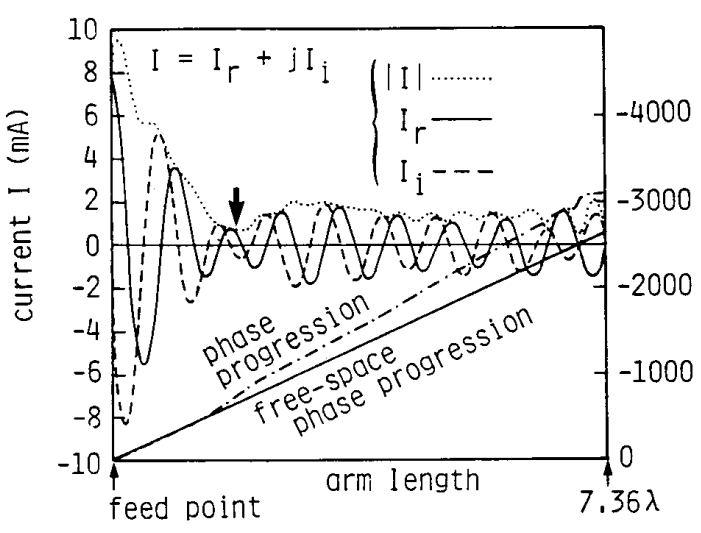

(a)

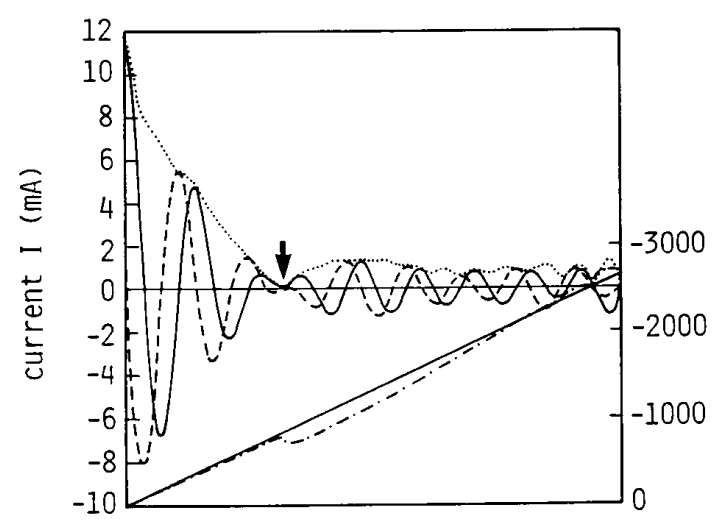

(b)

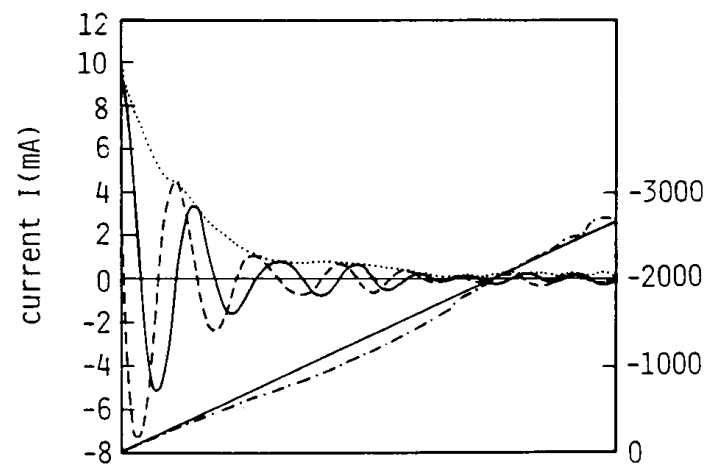

(c)
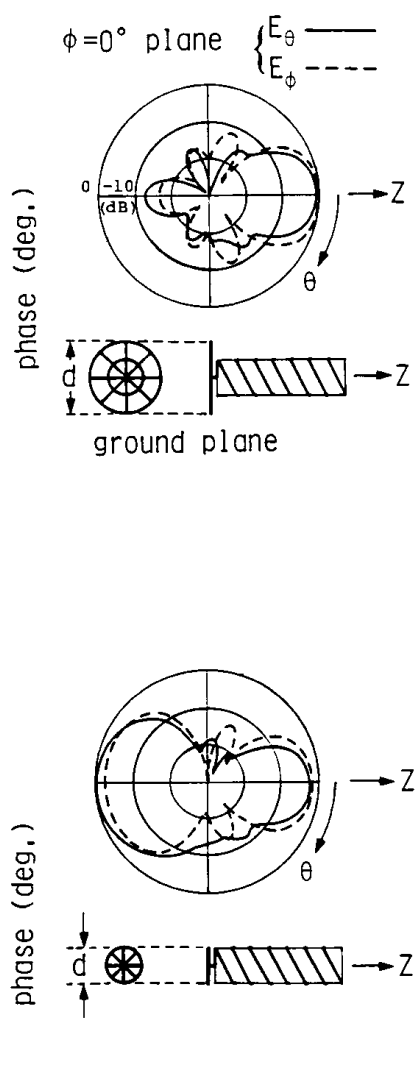

current distribution and radiation pattern. (a) $d=0.60 \lambda$. (b) $d=0.35 \lambda$

Fig. 2. Effects of wire-grid ground plane diameter on the current distribution
(c) $d=0.29 \lambda$

(= velocity on the helical wire/velocity of light in free space) on the surface wave region approximately satisfies the inphase condition of the forward endfire radiation. It follows that the radiation shows its maximum in the $+Z$-axis direction, where the power gain is calculated to be $11.6 \mathrm{~dB}$.

We should note that the current behavior and the radiation characteristics are similar to those of a helical antenna with an infinite ground plane [3], [4]. There are no inconsistencies with the empirical results that a helical antenna radiating in the forward endfire mode requires a ground plane whose diameter is more than $0.5 \lambda$.

As the diameter of the ground plane is decreased, the backward radiation becomes larger, as shown in Fig. 2(b), where the diameter is $d=0.35 \lambda$. Although the current distribution is similar to the previous one shown in Fig. 2(a), its amplitude on the surface wave region decreases and the first minimum shifts toward the arm end. This leads to the fact that the contribution of the decaying region to the radiation, i.e., 
backward radiation, becomes comparable to that of the surface wave region, i.e., the forward radiation.

After the progress described above, the helical antenna operates in the backfire mode. Fig. 2(c) is a typical example of the backfire mode, where the diameter of the ground plane is $d$ $=0.29 \lambda$ (the circumference of the ground plane is slightly smaller than that of the helical cylinder). The current distribution consists only of a decaying current, and its relative phase velocity is found to be nearly equal to 1.0 , which approximately satisfies the in-phase condition of the backfire radiation [13].

Further calculation shows that the radiation fields are circularly polarized with axial ratios of less than $3 \mathrm{~dB}$ both for the forward endfire mode (Fig. 2(a)) and the backfire mode (Fig. 2(c)). It is noted that the rotational sense of circular polarization of the forward endfire mode is the opposite of that of the backfire mode because of the antenna geometry and the direction of the propagating radiation field.

\section{Frequency Characteristics of Backfire Helical ANTENNA}

It has been found that a monofilar helical antenna operates in a backfire mode, when the ground plane is slightly smaller than that of the helix proper. The backfire-mode helix has an advantage of a small cross section because of a reduced ground plane. In this section we concentrate our attention on a backfire-mode helix and fully investigate its radiation characteristics. The parameters are as follows: The circumference of helical cylinder is $C=2.5 \mathrm{~cm}$, which is one wavelength at 12 $\mathrm{GHz}$; the ground plane diameter is $d=7.2 \mathrm{~mm}$; the number of helical turns and wire radius are $n=7$ and $\rho=0.3 \mathrm{~mm}$, respectively; and the pitch angle $\alpha$ is taken to be in a range of $6^{\circ}$ to $18^{\circ}$.

Figs. 3(a) and 3(b) show the frequency characteristics of the current distribution along the helical wire. Since the current form is not significantly affected by the change in the pitch angle, only the results for a pitch angle of $\alpha=14^{\circ}$ are presented. It is easily found that the current is dominated by a traveling wave with gradual attenuation. At lower frequencies the standing wave appears because of the insufficient attenuation of the traveling wave. This means that the attentuation rate determines the lower limit of an operating frequency. On the other hand, the upper limit of an operating frequency is determined by the deviation of the current phase from the inphase condition of the backfire radiation. The deviation of the current phase results in the deterioration of the front-to-back $(\mathrm{F} / \mathrm{B})$ ratio.

In general, the decaying current observed at intermediate frequencies contributes to the backfire radiation. If the decaying current which deviates from the in-phase condition for the backfire radiation appears and causes the forwardfire radiation, then a surface wave is generated as in the case of the axial mode helix. This fact is seen at higher frequencies, for instance, at $13 \mathrm{GHz}$, as shown in Fig. 3(b). At lower frequencies the surface wave is not appreciably excited, since the in-phase condition for the backfire radiation is approximately satisfied and the circumference of the helix is not large enough to support the surface wave [14].
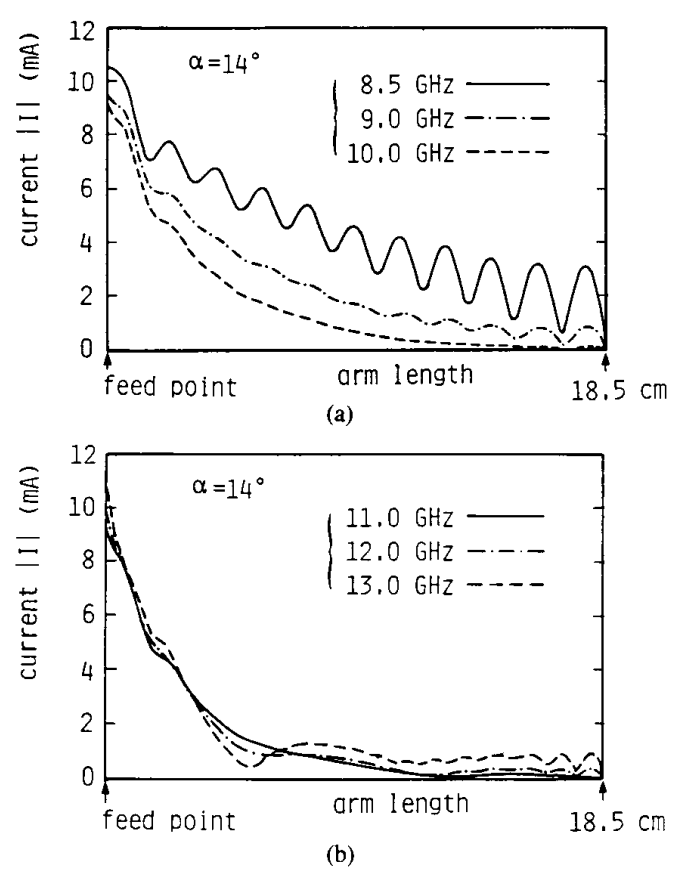

Fig. 3. Current amplitude as a function of frequency.

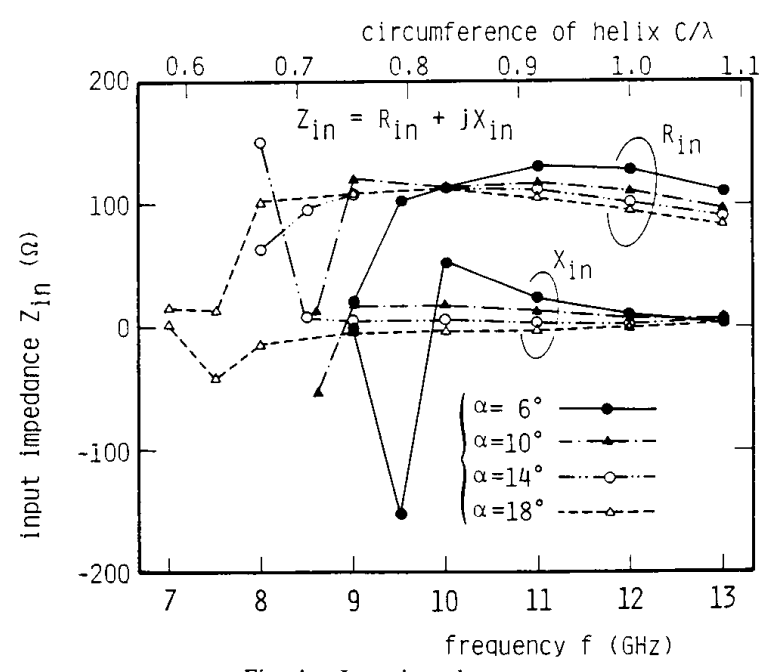

Fig. 4. Input impedance.

The input impedance calculated from the value of the current at the input is shown in Fig. 4. The input impedance shows a wide-band characteristic. Although the frequency range with a constant value depends on the pitch angle, the input impedance is of the order of $100 \mathrm{ohms}$ with a nearly pure resistance. (The input impedance of the helix can be matched to the characteristic impedance of a coaxial transmission line by changing the distance between the helix proper and the ground plane. We confirmed experimentally that a voltage standing-wave ratio (VSWR) of less than 1.1 can be realized by this technique.)

Fig. 5 shows the typical radiation patterns for a pitch angle of $\alpha=14^{\circ}$. In spite of the configuration of the helix being not 


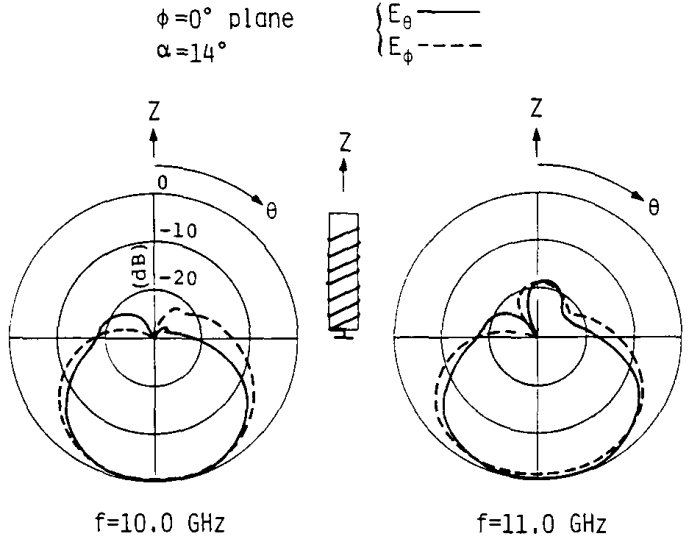

Fig. 5. Radiation pattern.

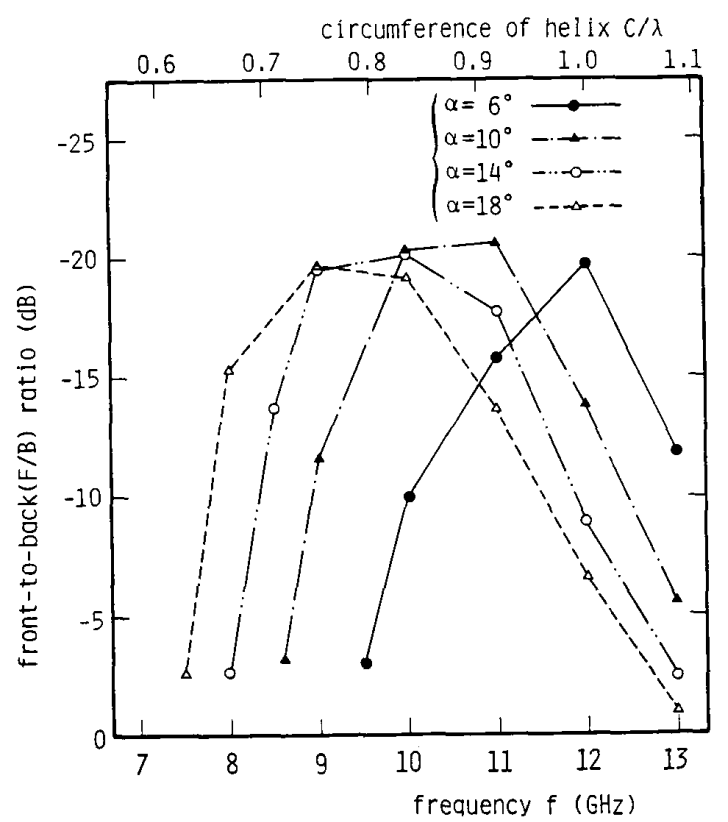

Fig. 6. Front-to-back ratio.

symmetrical with respect to the $Z$-axis, the radiation patterns maintain fairly good symmetry. The data concerning the radiation field are presented only for $\phi=0^{\circ}$ (similar results are obtained in other plane cuts).

The frequency dependence of the F/B ratio as a function of the pitch angle is shown in Fig. 6. The F/B ratio is evaluated by the average of $E_{\theta}$ and $E_{\phi}$ components of the radiation field. It is observed that the helix operates in the backfire mode over a wide range of frequencies. Calculation shows that the largest $\mathrm{F} / \mathrm{B}$ ratio is about $-20 \mathrm{~dB}$ for each pitch angle, and that the frequency range of operation in the backfire mode shifts toward higher frequencies as the pitch angle is decreased. As described earlier, the deterioration of the $\mathrm{F} / \mathrm{B}$ ratio at higher frequencies is caused by the deviation of the current phase from the in-phase condition for the backfire radiation, with subsequent excitation of the surface wave.

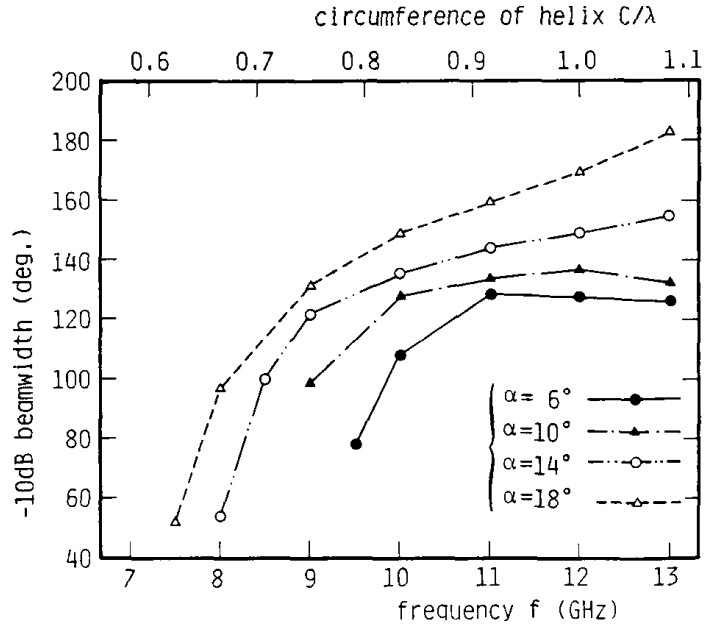

Fig. 7. $-10 \mathrm{~dB}$ beamwidth.

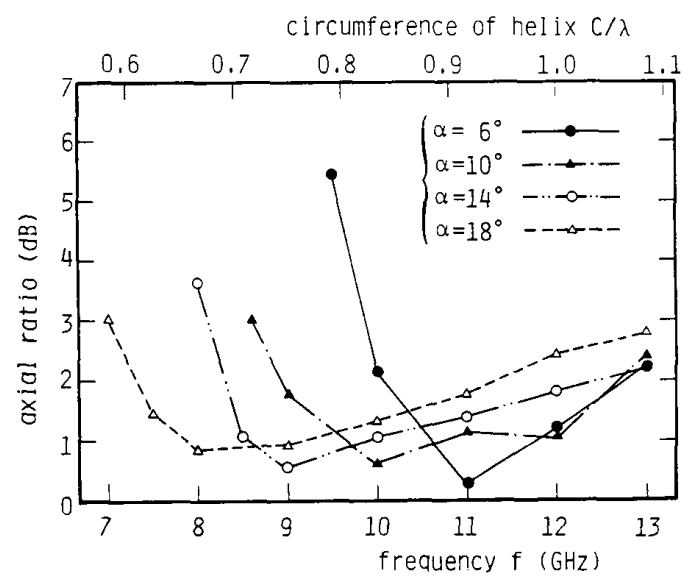

Fig. 8. Axial ratio.

In an application of the helix to a primary feed of a paraboloidal reflector, it is important to know the $-10 \mathrm{~dB}$ beamwidth [15] of the helix. Fig. 7 shows the frequency dependence of the $-10 \mathrm{~dB}$ beamwidth as a function of the pitch angle. It is seen that the beamwidth tends to widen as the frequency is raised (or the circumference of the helix is increased). However, the range of the beamwidth which can be controlled by the circumference is limited. It should be noted that the beamwidth can also be controlled by the pitch angle. The beamwidth is found to widen with an increase in pitch angle. For example, at $12 \mathrm{GHz}$, the $-10 \mathrm{~dB}$ beamwidth increases from $128^{\circ}$ to $170^{\circ}$, as the pitch angle is increased from $6^{\circ}$ to $18^{\circ}$. We can choose a desirable value from Fig. 7 , once the dimensions of the reflector are specified.

The calculated results concerning the axial ratio shown in Fig. 8 reveal that a circularly polarized wave (CPW) with an axial ratio of less than $3 \mathrm{~dB}$ is obtained over a wide frequency range of more than 1 to 1.3 . A lower limit frequency at which the helix operates as a CPW radiator tends to be lower as the pitch angle is increased.

Fig. 9 shows the power gain characteristic. For the most 


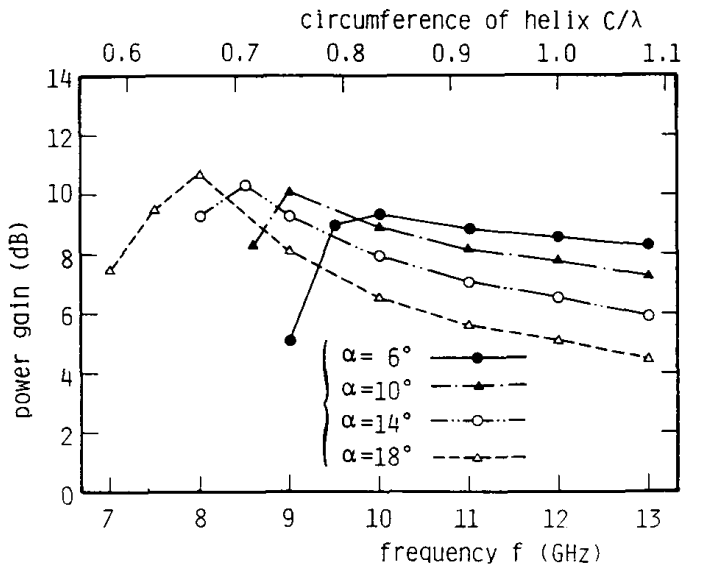

Fig. 9. Power gain.

part, the gain becomes gradually large as the frequency is decreased. This behavior is the same as that observed in a bifilar backfire helical antenna [16]. It is also noted that at frequencies of more than $10 \mathrm{GHz}$ the gain shows higher values for lower pitch angles. The gain is in a range of 4 to $10 \mathrm{~dB}$.

\section{Paraboloidal Reflector Antenna Fed By Monofilar BaCKfire Helix}

Now we employ the monofilar backfire helix as a primary feed of a symmetrical front-fed paraboloidal reflector. The reflector to be considered here has an aperture diameter of $D$ $=75 \mathrm{~cm}$ and a focal length of $L=22 \mathrm{~cm}$. The backfire helix is suitable for a feed of a front-fed reflector, since the configuration of the backfire helix is very small compared with a horn antenna and a conventional axial-mode helix with a ground plane. In addition, the backfire helix has an advantage that the feed line can be placed on the focal axis, resulting in a small aperture blockage.

To use the helix as an efficient feed for the reflector, it is imperative that the phase center location be known. The phase center is found by plotting the phase change of the radiation field against $\cos \theta$ [17]. The distance $s$ from the helix origin to the phase center is shown in Fig. 10. Strictly speaking, the distance $s$ varies according to the plane cut. Fig. 10 presents the average of the two distances obtained in $\phi=0^{\circ}$ and $90^{\circ}$ planes. As the frequency is increased, the distance $s$ moves toward the feed point. This is due to the fact that the attenuation rate of the current becomes larger with an increase in frequency, as shown in Fig. 3. The change in the distance $s$ as a function of frequency is small for a helix with a lower pitch angle, because the wire of the lower pitch helix is wound more tightly.

Fig. 11 shows the radiation pattern of the paraboloidal reflector antenna. A seven-turn backfire helix with a pitch angle of $\alpha=18^{\circ}$ and a ground plane diameter of $7.2 \mathrm{~mm}$ is situated at the focal point on the basis of the results of Fig. 10 . The pattern calculation is done at $12 \mathrm{GHz}$ using the physical optics approximation. It is seen that the half-power beamwidth is $2.4^{\circ}$ and that the first sidelobe level is less than $-26 \mathrm{~dB}$. The radiation field is circularly polarized with an axial ratio of $2.0 \mathrm{~dB}$. The power gain is calculated to be $37.2 \mathrm{~dB}$.

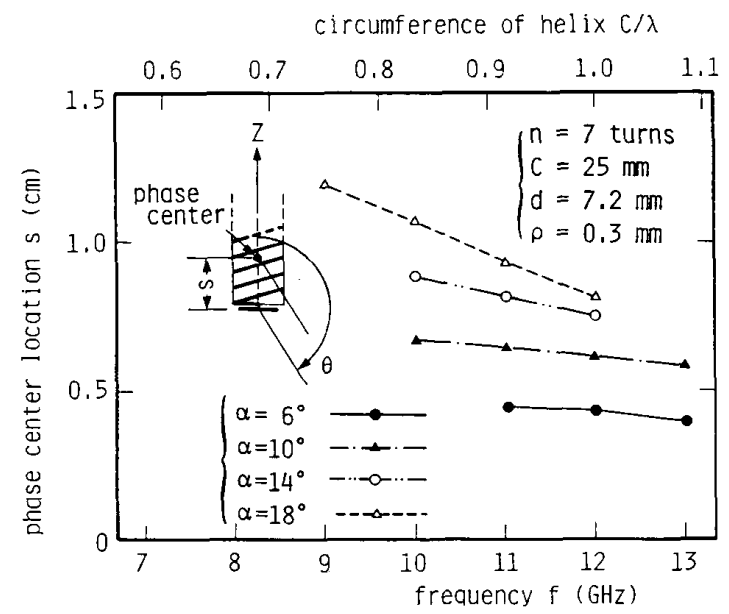

Fig. 10. Phase center location.

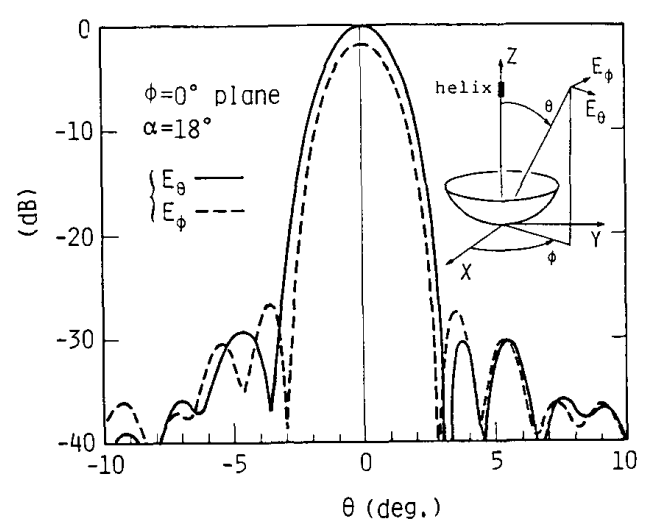

Fig. 11. Radiation pattern of paraboloidal reflector antenna fed by monofilar backfire helix.

\section{Conclusion}

The effects of a finite wire-grid ground plane of a monofilar helical antenna on the radiation characteristics have systematically been investigated by using a numerical method. As the diameter of the ground plane is decreased, a decaying current distribution with a relative phase velocity of about 1.0 becomes dominant, contributing to the backward radiation.

Subsequently, the backfire radiation of a monofilar helical antenna has been fully researched. It is found that the halfpower beamwidth can be controlled by not only the circumference of the helical cylinder but also the pitch angle. Calculation also shows that the radiation field is circularly polarized over a frequency range of more than 1 to 1.3 .

The last part of this paper has referred to the analysis of a paraboloidal reflector antenna fed by a monofilar backfire helix. The calculation at $12 \mathrm{GHz}$, based on the physical optics approximation, indicates a power gain of $37.2 \mathrm{~dB}$ for an aperture diameter of $75 \mathrm{~cm}$.

\section{ACKNOWLEDGMENT}

We wish to express our appreciation to Mr. A. Yoshizawa and Mr. Y. Kojima for their cooperation in writing a computer program. 


\section{REFERENCES}

[1] J. D, Kraus, Antennas. New York: McGraw-Hill, 1947.

[2] H. E. King and J. L. Wong, "Characteristics of 1 to 8 wavelength uniform helical antennas," IEEE Trans. Antennas Propagat., vol. AP-28, pp. 291-296, 1980

[3] H. Nakano and J. Yamauchi, "Characteristics of modified spiral and helical antennas," Proc. Inst. Elec. Eng., pt. H, vol. 129, pp. 232$237,1982$.

[4] H. Nakano, Y. Samada, and J. Yamauchi, "Axial mode helical antennas," IEEE Trans. Antennas Propagat., vol. AP-34, pp. 1143$1148,1986$.

[5] R. Pérez Leal and M. F. Cátedra, "Helical antennas: Influence of geometrical parameters on radiation characteristics," in 1983 Inst. Elec. Eng. Int. Conf. Antennas Propagat., pp. 395-398.

[6] W. T. Patton, "The backfire bifilar helical antenna," Univ. Illinois, Antenna Lab. Tech. Rep. 61, 1962.

[7] T. L. Sexson, "The backfire helical antenna," Sylvania Electron. Syst. Rep., ASTIA Doc. AD 626088, June 1965

[8] Y. Hashimoto, N. Misawa, K. Ishino, H. Nakano, M. Ando and N. Goto, "A DBS receiving parabolic antenna using a backfire helical," in 1987 Inst. Elec. Eng. Int. Conf. Antennas Propagat., pp. 133-136.

[9] H. E. King and J. L. Wong, " $240-400 \mathrm{MHz}$ antenna system for the fleetsatcom satellites," in 1977 IEEE Int. Antennas Propagat. Soc. Symp. Dig., pp. 349-352.

[10] R. C. Johnson and R. B. Cotton, "A backfire helical feed," IEEE Trans. Antennas Propagat., vol. AP-32, pp. 1126-1128, 1984.

[11] W. L. Stutzman and G. A. Thiele, Antenna Theory and Design. New York: Wiley, 1981

[12] H. Nakano, "Helical and spiral antennas-a numerical approach," Res. studies press, Wiley, 1987.

[13] H. Nakano, H. Mimaki, and J. Yamauchi, "Numerical analysis of a helical antenna with a finite ground plane," in 1986 IEEE Int. Antennas Propagat. Soc. Symp. Dig., pp. 129-132.

[14] H. Nakano, T. Yamane and J. Yamauchi, "Directive properties of parasitic helix and its application to circularly polarised antenna," Proc. Inst. Elec. Eng., pt. H, vol, 130, pp. 391-396, 1983.

[15] S. Silver, "Microwave antenna theory and design," M.I.T. Radiation Lab. Series, vol. 12. New York: McGraw-Hill, p. 427, 1949.

[16] H. Nakano, S. Iio and J. Yamauchi, "Frequency characteristics of tapered backfire helical antenna with loaded termination," Proc. Inst. Elec. Eng., pt. H, vol. 131, pp. 147-152, 1984.

[17] Y. Y. Hu, "A method of determining phase centers and its application to electromagnetic horns," J. Franklin Inst., vol. 271, pp. 31-39, 1961

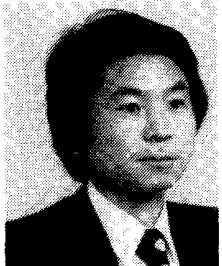

Hisamatsu Nakano (M'75-SM'87) was born in Ibaraki, Japan, on April 13, 1945. He received the B.E., M.E., and Dr.E. degrees in electrical engineering from Hosei University, Tokyo, in 1968, 1970 , and 1974 , respectively.

Since 1973, he has been a member of the Faculty of Hosei University, where he is now a Professor of Electrical Engineering. His research interests are numerical methods for microwave antennas and scattering problems. In 1981 he was a Visiting Professor at Syracuse University, NY, and contributed to formulating an electromagnetic coupling between a wire and a slot. In 1986 and 1987 he was a Visiting Professor at the University of Manitoba, Winnipeg, and the University of California, Los Angeles, respectively. During his stay in Canada, he received the international Scientific Exchange Award. In 1987 he received the Best Paper Award of the IEE Fifth International Conference on Antennas and Propagation.

Dr. Nakano is a member of the Institute of Electronics, Information and Communication Engineers of Japan.

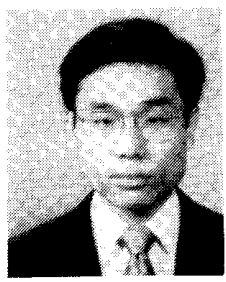

Junji Yamauchi (M'85) was born in Nagoya, Japan, on August 23, 1953. He received the B.E., M.E., and Dr. E. degrees from Hosei University, Tokyo, in 1976, 1978 and 1982, respectively.

From 1984 to 1988 , he served as a Lecturer at the Electrical Engineering Department of Tokyo Metropolitan Technical College. Since 1988, he has been a member of the Faculty of Hosei University, where he is a Lecturer of Electrical Engineering. His research interests are in circularly polarized antennas and waveguides.

Dr. Yamauchi is a member of the Institute of Electronics, Information and Communication Engineers of Japan.

Hiroaki Mimaki, for a photograph and biography please see page 796 of the June 1986 issue of this TRANSACTIONS. 